\title{
Turbidimetric Determination of Particle Size Distributions of Colloidal Systems
}

\author{
D. H. MELIK AND H. S. FOGLER \\ Department of Chemical Engineering, The University of Michigan, Ann Arbor, Michigan 48109
}

Received March 8, 1982; accepted July 7, 1982

\begin{abstract}
Application of the Mie theory of light scattering to measurements of the turbidity ratio and the wavelength exponent provides an easy method for estimating particle size distributions of nonabsorbing isotropic spheres in the micrometer to submicrometer range. Combining both these lightscattering techniques not only allows one to analyze particle sizes which are too large for quasi-elastic light scattering and too small for optical microscopy, but can be accomplished with only two turbidity measurements and no prior knowledge of the particle volume fraction. An algorithm is presented for constructing turbidity spectra, for any system of known optical constants and known distributional form, which can be used to easily determine the mean diameter and standard deviation of an unknown distribution. Using this algorithm, size-distribution curves were obtained from turbidity measurements at two widely separated wavelengths. These distributions are in agreement with distributions determined from scanning electron microscopic analysis.
\end{abstract}

\section{INTRODUCTION}

The Mie theory of light scattering offers an attractive means of studying dispersions of fine particles. The principal advantages of light scattering as a method of particle size analysis include:

(a) Measurements are rapid and simple.

(b) Sampling problems are minimized since the method is employed in situ.

(c) Measurement causes no disturbance of the system which is simply illuminated with a beam of light.

(d) Independence of the system being studied; it does not matter whether the distribution of particle sizes was altered by creaming or flocculation, or from their combined effects.

Many investigators (1-8) have developed various turbidity techniques to determine the polydispersity of colloidal suspensions. The particle size distributions estimated from these turbidimetric methods were found to be in good agreement with other techniques, such as the scanning electron microscope and the Coulter counter. In each case, either a measurement of the particle volume fraction was required along with two turbidity readings, or turbidity measurements from at least three wavelengths were necessary. A turbidity technique has not been developed to estimate the particle size distribution of systems with an unknown volume fraction from only two turbidity readings. A technique of this type would be extremely useful in determining size-distribution curves of flocculating emulsions during centrifugation since:

(a) Previous ultracentrifuge data analysis methods have not accounted for the formation of aggregates (9).

(b) The volume fraction continually decreases during centrifugation, and consequently remains unknown after the initial start-up.

(c) Only two turbidity measurements can be taken simultaneously on a conventional ultracentrifuge without upgrading the system with expensive electronic hardware.

This paper describes a turbidimetric method for determining the particle size distribution from only two turbidity measure- 
ments. By combining two light-scattering parameters, the turbidity ratio and the rate at which turbidity changes with wavelength (called the wavelength exponent), either a one- or a two-parameter distribution can be estimated if the form of the distribution is known or can be assumed. The first part of this paper is a fundamental review of previous work, and is included for the sake of completeness.

In order to appraise the proposed turbidity technique, size-distribution curves estimated from this turbidimetric method are compared with distributions obtained from scanning electron micrographs.

\section{THEORY}

\subsection{Turbidity}

The turbidity, $\tau$, of a suspension of particles is a measure of the reduction in intensity of the transmitted beam due to scattering. The turbidity is defined by (10)

$$
\tau=\frac{1}{l} \ln \left[\frac{I_{0}}{I}\right],
$$

where $l$ is the scattering path length and, $I_{0}$ and $I$ are the intensities of the incident and transmitted beams, respectively. In terms of the optical density $D$ of the suspension

$$
\tau=2.303 \mathrm{D} / \mathrm{l} \text {. }
$$

For a monodisperse system of nonabsorbing isotropic spheres in the absence of multiple and dependent scattering the turbidity is given by (10)

$$
\tau=\pi N r^{2} Q(r, \lambda, m),
$$

where $\tau$ is a function of the particle concentration, $N$, the particle radius, $r$, and the scattering coefficient, $Q$, which is defined as the ratio of the scattering to geometrical cross sections. The scattering cross section is the total radiation scattered by a single sphere from an incident beam of unit intensity. This scattering coefficient is, in turn, a function of the particle size, the ratio of the refractive index of the particles $\mu_{\mathrm{p}}$ to the refractive in- dex of the medium $\mu_{m}$, denoted by $m$, and the wavelength of the incident wave in the dispersing medium, $\lambda$. The wavelength $\lambda$ is equal to $\lambda_{0} / \mu_{m}$, where $\lambda_{0}$ is the wavelength in air. The scattering coefficient is calculated using the Mie theory of light scattering and will be discussed later.

By introducing a dimensionless particle size $\alpha$

$$
\alpha=\frac{2 \pi r}{\lambda}
$$

the scattering coefficient becomes a function of $\alpha$ and $m$ only. Using this variable, Eq. [3] is rewritten to give

$$
\tau=\frac{N \alpha^{2} \lambda^{2}}{4 \pi} Q(\alpha, m)
$$

Incorporating the volume fraction $\phi$ given by

$$
\phi=\frac{4 \pi r^{3}}{3} N=\frac{\alpha^{3} \lambda^{3}}{6 \pi^{2}} N
$$

into Eq. [5] and rearranging yields a dimensionless turbidity

$$
\frac{\tau \lambda}{\phi}=\frac{3 \pi}{2} \frac{Q(\alpha, m)}{\alpha} .
$$

In Fig. 1, the dimensionless turbidity for $m=1.10,1.15$ is plotted up to $\alpha=26.0$. This figure covers the range of colloidal sizes that are most likely to be investigated by light

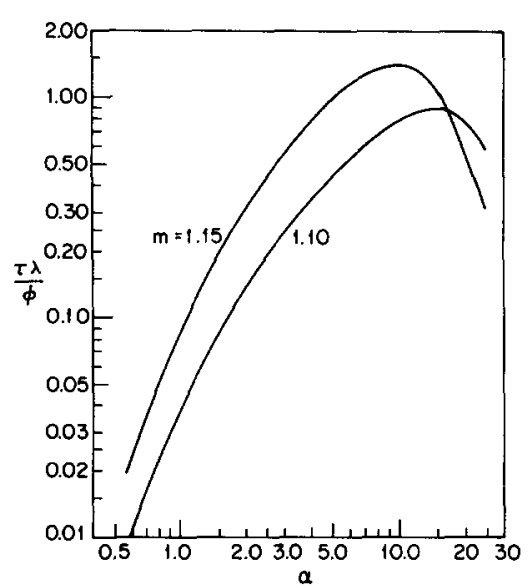

FIG. 1. Dimensionless turbidity of a monodisperse system. 
scattering, and is applicable up to particle radii of $2.0 \mu \mathrm{m}$ for green light in water. For this range the multivaluedness is reduced to a possibility of two values. Measurements at two wavelengths allow one to decide unambiguously whether one is operating on the ascending or descending branch of these curves, and by comparison with the theoretical curve, the particle size can be obtained.

\subsection{Wavelength Exponent}

This method makes use of the dependence of the turbidity on the wavelength of the incident beam. This wavelength effect is influenced most directly through the variation of $Q(\alpha, m)$ with $\alpha$ and, more subtly, by the dispersion of the refractive index with wavelength.

For a monodisperse system of nonabsorbing isotropic spheres in the absence of multiple and dependent scattering, the exponent $g$, associated with $\lambda_{0}$, is given by (11)

$$
\frac{\tau}{\phi}=k \lambda_{0}^{-g},
$$

where

$$
k=\frac{3 \pi \mu_{m}}{2} \frac{\lambda_{0}^{g-1}}{\alpha} Q(\alpha, m) .
$$

For any given $m, \mu_{m}$, and $\lambda_{0}$, the wavelength exponent will have a value of 4 for Rayleigh scatterers $(2 \alpha[m-1] \ll 1)$, will decline with increasing particle size until it reaches zero, and will subsequently assume negative values. As long as the wavelength exponent remains single valued, it can be used to determine particle sizes.

To derive the manner in which the turbidity changes with wavelength, one takes the negative of the derivative of $\ln (\tau / \phi)$ with respect to $\ln \lambda_{0}$, at a constant value of the particle size $\alpha$. This gives

$$
-\frac{d \ln (\tau / \phi)}{d \ln \lambda_{0}}=g-\frac{d \ln k}{d \ln \lambda_{0}}=n_{0},
$$

where the experimental exponent $n_{0}$ is obtained from the slope, at a selected wavelength, of a $\ln (\tau / \phi)$ versus $\ln \lambda_{0}$ plot. If only an approximate particle size is desired, $n_{0}$ could be used in place of $g$ in Eq. [8] (12). In order to translate the exponent obtained experimentally into accurate particle size determinations, it is necessary to evaluate $n_{0}$ as a function of $\alpha$ and $m$ using the Mie scattering theory.

Since $\mu_{m}, m$, and $\alpha$ are functions of $\lambda_{0}$, inserting Eq. [7] into Eq. [10] gives

$$
n_{0}=n(\alpha, m) F_{1}-P(\alpha, m) F_{2},
$$

where

$$
\begin{aligned}
n(\alpha, m) & =\left[\frac{\partial \ln Q(\alpha, m)}{\partial \ln \alpha}\right]_{m}, \\
P(\alpha, m) & =\left[\frac{\partial \ln Q(\alpha, m)}{\partial m}\right]_{\alpha}, \\
F_{1} & =1-\left.\frac{d \ln \mu_{m}}{d \ln \lambda_{0}}\right|_{\lambda_{0}},
\end{aligned}
$$

and

$$
F_{2}=\left.\frac{d m}{d \ln \lambda_{0}}\right|_{\lambda_{0}} .
$$

The correction terms $F_{1}$ and $F_{2}$ depend on the optical properties of the particles and the medium (analytical expressions for $F_{1}$ and $F_{2}$ are given in Appendix B); while $n(\alpha, m)$ and $P(\alpha, m)$ can be calculated from theoretical $Q(\alpha, m)$ values. These calculations will be considered later.

The wavelength exponent $n_{0}$ is plotted in Fig. 2 for various values of the particle size $\alpha$. A plot of this type enables a determination

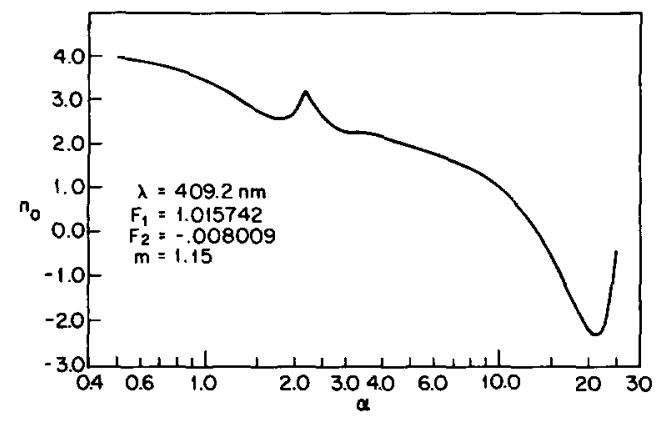

FIG. 2. Wavelength exponent of a monodisperse system for incident light of $546.1 \mathrm{~nm}$ in a water medium at $25^{\circ} \mathrm{C}$.

Journal of Colloid and Interface Science, Vol. 92, No. 1, March 1983 
of the particle size parameter from a measurement of the experimental exponent at $\lambda_{0}=546.1 \mathrm{~nm}$ for systems in a water medium (i.e., $\mu_{m}=1.33$ ). For other wavelengths, similar plots need to be constructed.

\subsection{Polydispersions}

The above development is applicable only for dispersions of uniform particle size. We now turn to the problem of determining the distribution of particle sizes by turbidity when the spheres comprising the dispersion are no longer of nearly the same size.

Since the turbidity of a polydispersion is the sum of all the contributions over the various particle sizes, the turbidity of the total distribution expressed in integral form is (10)

$$
\tau=\frac{N \lambda^{2}}{4 \pi} \int_{0}^{\infty} \alpha^{2} f(\alpha) Q(\alpha, m) d \alpha,
$$

where $f(\alpha) d \alpha$ is the number fraction of particles with a size between $\alpha$ and $\alpha+d \alpha$. Equation [16] can be put into a more tractable form by incorporating the volume fraction $\phi$. The volume fraction of the particles in the suspending medium is related to the particle concentration by

$$
\phi=\frac{4 \pi N}{3} \int_{0}^{\infty} r^{3} f(r) d r .
$$

Since $f(\alpha) d \alpha=f(r) d r$, in terms of the particle size parameter $\alpha$ this becomes

$$
\phi=\frac{N \lambda^{3}}{6 \pi^{2}} \int_{0}^{\infty} \alpha^{3} f(\alpha) d \alpha
$$

Inserting Eq. [18] into Eq. [16] and rearranging gives a dimensionless turbidity

$$
\frac{\tau \lambda}{\phi}=\frac{3 \pi}{2} \frac{\int_{0}^{\infty} \alpha^{2} f(\alpha) Q(\alpha, m) d \alpha}{\int_{0}^{\infty} \alpha^{3} f(\alpha) d \alpha} .
$$

Since, in general, the volume fraction is either known only approximately or not at all, it can be eliminated by taking a ratio of turbidities at two widely separated wavelengths

$$
\begin{array}{r}
\frac{(\tau \lambda)_{\lambda_{1}}}{(\tau \lambda)_{\lambda_{2}}}=\frac{\left[\int_{0}^{\infty} \alpha^{2} f(\alpha) Q(\alpha, m) d \alpha\right]_{\lambda_{1}}}{\left[\int_{0}^{\infty} \alpha^{2} f(\alpha) Q(\alpha, m) d \alpha\right]_{\lambda_{2}}} \\
\times \frac{\left[\int_{0}^{\infty} \alpha^{3} f(\alpha) d \alpha\right]_{\lambda_{2}}}{\left[\int_{0}^{\infty} \alpha^{3} f(\alpha) d \alpha\right]_{\lambda_{1}}}
\end{array}
$$

Equation [20] is known as the turbidity ratio for a polydisperse system of spherical particles. Knowing the distribution of particle sizes $f(\alpha)$, the expected turbidity ratio can be computed. However, the real problem lies in finding the unknown distribution from a series of turbidity ratio readings.

The corresponding wavelength exponent for a polydisperse system is obtained by substituting Eq. [19] into Eq. [10] to give an average exponent

$$
\begin{aligned}
& \left\{F_{1} \int_{0}^{\infty} \alpha^{2} f(\alpha) \frac{\partial Q(\alpha, m)}{\partial \ln \alpha} d \alpha\right. \\
& \bar{n}_{0}=\frac{\left.-F_{2} \int_{0}^{\infty} \alpha^{2} f(\alpha) \frac{\partial Q(\alpha, m)}{\partial m} d \alpha\right\}}{\int_{0}^{\infty} \alpha^{2} f(\alpha) Q(\alpha, m) d \alpha} .
\end{aligned}
$$

Incorporating the definitions of $n(\alpha, m)$ and $P(\alpha, m)$, given by Eqs. [12] and [13], respectively, the final form of the wavelength exponent becomes

$$
\begin{gathered}
\left\{F_{1} \int_{0}^{\infty} \alpha^{2} f(\alpha) Q(\alpha, m) n(\alpha, m) d \alpha\right. \\
\bar{n}_{0}=\frac{\left.-F_{2} \int_{0}^{\infty} \alpha^{2} f(\alpha) Q(\alpha, m) P(\alpha, m) d \alpha\right\}}{\int_{0}^{\infty} \alpha^{2} f(\alpha) Q(\alpha, m) d \alpha} .
\end{gathered}
$$


Just as in the case of the turbidity ratio, if the distribution of particle sizes is known, the expected wavelength exponent can be calculated. However, the objective is to use the experimentally observed exponent to obtain the unknown distribution.

\subsection{Calculation of $Q(\alpha, m), n(\alpha, m)$, and $P(\alpha, m)$}

The scattering coefficient $Q(\alpha, m)$ is generally computed from the rigorous Mie theory and can be expressed in the form (10)

$$
\begin{aligned}
Q(\alpha, m)=\frac{2}{\alpha^{2}} \sum_{n=1}^{\infty}(2 n & +1) \\
& \times\left\{\left|a_{n}\right|^{2}+\left|b_{n}\right|^{2}\right\},
\end{aligned}
$$

where $a_{n}$ and $b_{n}$ are complex functions of $\alpha$ and $m$. Except for small, $\alpha<0.6$, and large, $\alpha>25.0$, particles, Eq. [23] should be used to accurately calculate $Q$. Scattering coefficients for colloidal systems are tabulated quite extensively in the literature (13-15). For small and large particles, approximation formulas, which are accurate within at least $1 \%$ of the values calculated from Eq. [23], can be used without any major loss of accuracy. For $\alpha<0.6$, the approximate relation of Schoenberg and Jung can be used (16)

$$
\begin{aligned}
Q=\frac{8}{3} \alpha^{4}\left[\frac{m^{2}-1}{m^{2}+2}\right]^{2} & \\
& \times\left\{1+\frac{6}{5} \alpha^{2}\left[\frac{m^{2}-2}{m^{2}+2}\right]\right\} .
\end{aligned}
$$

For $\alpha>25.0$ and $1<m \leq 1.25$, the equation of Van de Hulst can be applied (16)

$$
\begin{array}{r}
Q=2-\frac{16 m^{2} \sin \rho}{(m+1)^{2} \rho}+\frac{4(1-m \cos \rho)}{\rho^{2}} \\
+7.53 \frac{(z-m)}{(z+m)} \alpha^{-.772},
\end{array}
$$

where

and

$$
\rho=2 \alpha(m-1),
$$

$$
z=\left[\left(m^{2}-1\right)(6 \alpha / \pi)^{2 / 3}+1\right]^{1 / 2} \text {. }
$$

(Note: Van de Hulst's approximation is actually correct within $1 \%$ for $\rho>2.4$, but in this work is used only for $\alpha>25.0$.) The light scattering coefficient $Q(\alpha, m)$ is plotted in Fig. 3 for various values of the particle size $\rho$.

Equation [12] is used to calculate $n(\alpha, m)$ and Eq. [13] to calculate $P(\alpha, m)$. For $\alpha$ $<0.6$ and $\alpha>25.0$, analytical formulas are derived from Eqs. [24] and [25]. For intermediate $\alpha$ values, numerical differentiation is employed to calculate the derivatives from tabulated $Q(\alpha, m)$ values. A central derivative formula was selected as the most accurate method for deriving $n(\alpha, m)$ and $P(\alpha, m)$ (17)

$$
\begin{aligned}
\frac{d y}{d x}=(1 / 60 h) & {\left[45\left(y_{1}-y_{-1}\right)\right.} \\
& \left.-9\left(y_{2}-y_{-2}\right)+\left(y_{3}-y_{-3}\right)\right]
\end{aligned}
$$

where $h$ is the constant interval between successive $\alpha$ values for $n(\alpha, m)$ or successive $m$ values for $P(\alpha, m)$. The quantities $y_{\mathrm{u}}$ and $y_{-\mathrm{u}}$ are the $\ln Q(\alpha, m)$ values pertinent to the $\alpha$ or the $m$ values which follow and precede, respectively, by $u$ unit intervals, the $\alpha$ or the $m$ value to be considered.

\section{DEVIATIONS FROM IDEALITY}

Since it is difficult to choose experimental conditions which are in agreement with the assumptions underlying scattering theory, it

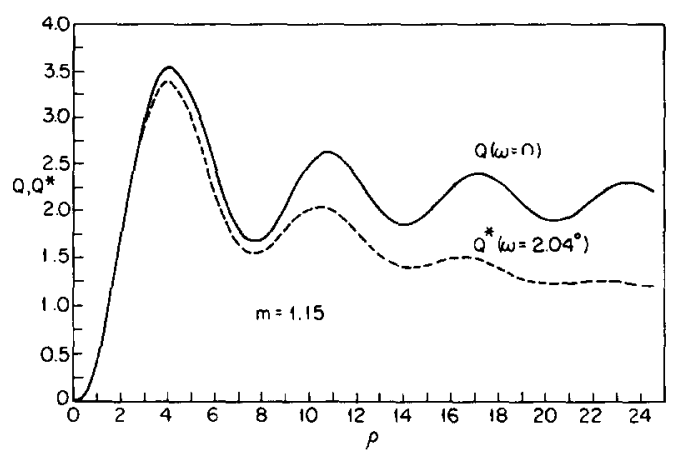

Fig. 3. Values of the light scattering coefficient $Q$, and of the corrected coefficient, $Q^{*}$, for the case where $\omega$ $=2.04^{\circ}$. 
is usually necessary to apply a number of corrections. A detailed discussion of the possible errors involved in turbidimetry is given elsewhere $(18,19)$; however, the more important errors and corrections relative to this work will be reviewed, and the instrumental requirements discussed.

\subsection{Instrumentation}

One notes from Eq. [1] that a light-transmission optical system (transmissometer) must measure the ratio $I_{0} / I$ in order to determine the turbidity $\tau$. A primary concern here is that $I$ is the light energy which has traveled the path length $l$ and which has not been scattered. This implies that an ideal instrument will not measure any of the light that is scattered. However, since scattering occurs in all directions, no such instrument exists. All transmissometers will accept scattered light to some degree; consequently, some error results.

The effect of scattered light on turbidity measurements is characterized by a "finite angle of acceptance," $\omega(20)$. The angle $\omega$ is equal to one-half the sum of the divergence or convergence of the primary beam, $2 \theta_{\mathrm{s}}$, and the angle subtended by the detector with reference to the center of the scattering volume, $2 \theta_{\mathrm{d}}$. To the extent that $\omega$ is not zero, the detector will also be accepting any scattered light which falls within its field of view. This results in a measured turbidity $\tau^{*}$ which is less than the true turbidity $\tau$ by some amount $\delta$, which implies an error in the measurement of $I_{0} / I$. Therefore, the angle $\omega$ should be kept as small as possible. Moreover, secondary scattered light from outside the suspension volume which is illuminated by the incident beam should also be prevented from reaching the detector.

Of the various optical systems which have been used in light-transmission experiments, the lens-pinhole system shown in Fig. 4 is preferred for three reasons (21-23):

(a) The exact value for the detector's halfangle $\theta_{\mathrm{d}}$ can be calculated from a direct measurement of the diameter of the pinhole and the focal length of the lens. Referring to Fig. 4 , light from the monochromator $A$ is focused into a nearly parallel beam by lens $L_{1}$. Beam stop $B_{1}$ determines the diameter of the light beam, which then passes through the optical cell (cuvette) C. $B_{2}$ serves to eliminate secondary scattered light and lens $L_{2}$ focuses the beam at the pinhole, which is located at the focal length of this lens. The pinhole must be large enough to permit all the light in the focused beam to pass through and fall on the cathode of the phototube $P$. The value of $\theta_{\mathrm{d}}$ is equal to the arc tangent of $a / f$, where $a$ is the radius of the pinhole and $f$ the focal length of lens $\mathrm{L}_{2}$ (24).

(b) The value of $\theta_{\mathrm{d}}$ is constant and independent of the location of the illuminated particle in the path of the beam or in the fringes of the beam, so long as the diameter of lens $\mathrm{L}_{2}$ satisfies the condition

$$
D_{\mathrm{L}_{2}} \geq \frac{2 a z}{f}+D_{\mathrm{b}},
$$

where $D_{\mathrm{L}_{2}}$ is the diameter of lens $\mathrm{L}_{2}, z$ the distance between lens $L_{2}$ and the most remote particle in the beam, and $D_{\mathrm{b}}$ the di-

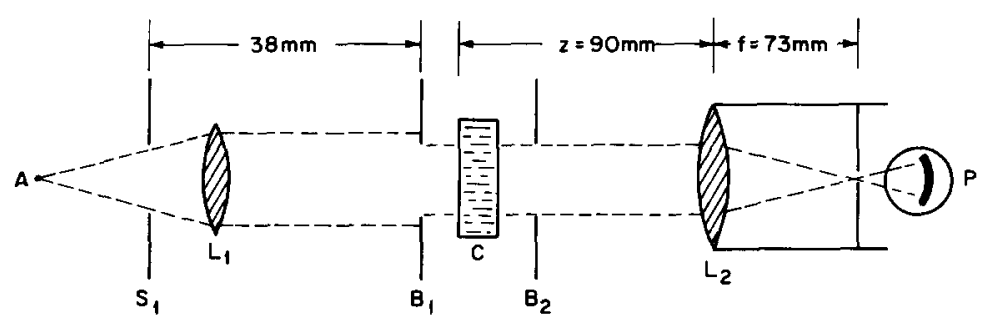

FIG. 4. Schematic of lens-pinhole optical system. 


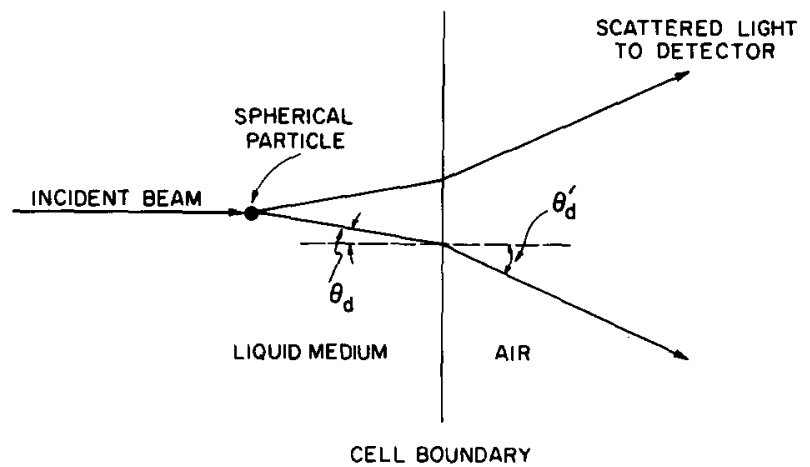

FIG. 5. Effect of dispersing medium on the half-angle of the detector.

ameter of the light beam as determined by beam stop $\mathbf{B}_{1}$.

(c) The lens-pinhole optical system practically excludes all stray light from the phototube.

When transmission measurements are being made on a liquid dispersion of immiscible particles, one must account for the deflection of the scattered light upon crossing the boundaries of the cell as shown in Fig. 5 (22). Applying Snell's law of refraction (25) to Fig. 5 , and noting that $a / f$ is usually quite small, the value of $\theta_{\mathrm{d}}$ for particles in a liquid medium is given by

$$
\theta_{\mathrm{d}}=\frac{\theta_{\mathrm{d}}^{\prime}}{\mu_{m}}
$$

where $\theta_{\mathrm{d}}^{\prime}$ is the angle measured by the ratio $a / f$, and $\mu_{m}$ is the refractive index of the medium.

In addition, since a point source is physically impossible, a perfectly collimated in- cident beam is not feasible. The light beam either slightly converges or diverges from a perfectly collimated path as shown in Fig. 6 . This effect can be corrected by measuring the half-angle of divergence or convergence, $\theta_{\mathrm{s}}$, also shown in Fig. 6, and adding this halfangle to the detector's to obtain the total finite angle of acceptance $\omega$.

The angular size of this divergence or convergence, $\theta_{\mathrm{s}}$, is determined from the dimensions of the exit slit $S_{1}$ and the dimensions of the light beam at a point downstream from slit $S_{1}$; for example, at beam stop $B_{1}$. (An example of this calculation is given in the experimental section.) Just as in the case of the detector's half-angle, one must account for the light deflected upon crossing the cell boundaries by using a relationship analogous to Eq. [30]. Finally, it should be pointed out that the detector's half-angle should be larger than the divergence or convergence of the primary beam to ensure that all of the beam energy will arrive at the phototube.

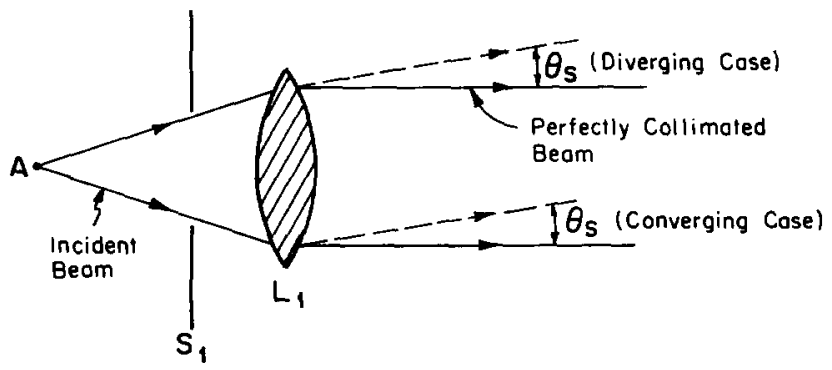

FiG. 6. Divergence or convergence of the primary beam. 


\subsection{Correcting for Scattered Light}

Even when $\omega$ is kept small the influence of scattered light on the turbidity may be important; it should be taken into account as soon as $\omega \alpha>0.2$ ( $\omega$ measured in radians). Fortunately, it suffices to apply a correction to $Q(\alpha, m)$, as long as $\omega$ is kept small $\left(\omega<5^{\circ}\right)$. The correction $C_{Q}$ for scattered radiation falling on the detector, to be subtracted from $Q$, is given by

$$
C_{Q}=\alpha^{-2} \int_{0}^{\omega}\left[i_{1}(\xi)+i_{2}(\xi)\right] \sin \xi d \xi,
$$

which is deduced from basic scattering theory $(10)$. The sum $\left[i_{1}(\xi)+i_{2}(\xi)\right]$ is the total intensity of light scattered in a direction defined by the angle $\xi$ when the incident wave is of unit intensity. The angle $\xi$ is the angle between the direction of observation and the direction of the primary beam. A detailed discussion on this scattered-light correction is given by Deepak and Box for both monodisperse (21) and polydisperse (26) systems. Unfortunately, calculation of $C_{Q}$ by means of the rigorous Mie theory is time consuming, and therefore it is worthwhile to use an approximation which yields sufficient accuracy. With the aid of the following approximation, Walstra (6) obtained significantly more accurate size-distribution curves than would be possible if this correction were neglected.

For $\rho>2.5$ and $1<m \leq 1.25$, the corrected scattering coefficient $Q^{*}$ can be found from the relation (19)

$Q^{*}(\alpha, m)=Q(\alpha, m)-F(\alpha, m) R(\omega \alpha)$,

where $Q(\alpha, m)$ is given by Eq. [23], [24], or [25],

$$
\begin{aligned}
F(\alpha, m)= & \left(B^{2}+Q^{2}\right) / 4.0, \\
R(\omega \alpha)= & 1-J_{0}^{2}(\omega \alpha)-J_{1}^{2}(\omega \alpha), \\
J_{0}, J_{1}= & \text { Bessel functions of the first kind } \\
& \text { of zero and first order, respec- } \\
& \text { tively, and }
\end{aligned}
$$

the term $B(\alpha, m)$ is given by (19)

$$
\begin{array}{r}
B(\alpha, m)=\frac{-16 m^{2} \cos \rho}{(m+1)^{2} \rho}+\frac{4 m \sin \rho}{\rho^{2}} \\
-\frac{4.2(z-m)}{(z+m)} \alpha^{-.772}
\end{array}
$$

where $\rho$ and $z$ are given by Eqs. [26] and [27], respectively. The corrected light-scattering coefficient $Q^{*}(\alpha, m)$ is plotted in Fig. 3 as a function of the particle size $\alpha$ for the experimental conditions discussed later (i.e., $\omega=2.04^{\circ}$ ).

It should be remarked that the turbidity is not a function of $Q$ but of $Q^{*}$. Therefore, $Q$ in Eqs. [3], [5], [7], [9], [12], [13], [16], [19], [20], [21], and [22] should be replaced with $Q^{*}$. Similarly, $n(\alpha, m)$ in Eqs. [11], [12], and [22] should be replaced with $n^{*}$, and $P(\alpha$, $m)$ in Eqs. [11], [13], and [22] should be replaced with $P^{*}$.

\subsection{Other Requirements}

In addition to forward scattering and secondary scattering, there are a series of other factors which may interfere with the accuracy of the theory as outlined here. The most frequent difficulty in turbidimetry is the failure of Beer's law: the optical density increases to a smaller extent than is proportional with concentration, thus necessitating extrapolation to zero concentration. As in absorption photometry, this may sometimes be caused by a large spectral bandwidth, but is usually due to other phenomena such as multiple and dependent scattering. Multiple scattering arises whenever the particles receive light scattered by other particles in addition to radiation from the incident beam; while dependent scattering arises whenever the particles are not randomly positioned, the overall interference between waves scattered by different particles is not zero. In order to avoid these deviations the following precautions should be taken: (i) $\omega$ should be at most 3 or 4 degrees; (ii) the optical density must not be larger than 0.7 or 0.8 (and preferably not smaller than 0.15 for reasons of accu- 
racy); and (iii) the volume fraction must never exceed 0.002 .

\section{DETERMINING UNKNOWN PARTICLE SIZE DISTRIBUTION}

In this section we will show how one can apply the theory developed in Sections 2 and 3 to determine the unknown distribution $f(\alpha)$ from measurements of the turbidity ratio and wavelength exponent. Since an important application of our proposed turbidity technique would be to estimate the distribution of particle sizes during centrifugation, we will work with the two visible wavelengths found on a conventional ultracentrifuge $\left(\lambda_{01}\right.$ $=435.8 \mathrm{~nm}, \lambda_{02}=546.1 \mathrm{~nm}$, or $\lambda_{1}=325.2$ $\mathrm{nm}, \lambda_{2}=409.2 \mathrm{~nm}$ in a water medium), even though other wavelengths can be used.

\subsection{Size-Distribution Functions}

As mentioned in Section 2.3, Eqs. [20] and [22] can be used to determine the unknown frequency distribution function $f(\alpha)$. However, these equations are extremely difficult to invert without assuming a distributional form a priori. Two common distributions found in colloid science are the two-parameter log-normal distribution and the one-parameter positively skewed distribution (27).

The log-normal distribution by number is given by

$$
\begin{aligned}
f(\alpha)= & \frac{q}{(2 \pi)^{1 / 2} \alpha} \\
& \times \exp \left[-\frac{q^{2}}{2}(\ln \alpha-\ln p)^{2}\right],
\end{aligned}
$$

where

$$
\begin{aligned}
q & =1 / \mathrm{ln} \sigma_{g}, \\
\sigma_{g} & =\text { geometric standard deviation, } \\
p & =2 \pi a_{m} / \lambda, \text { and } \\
a_{m} & =\text { geometric mean radius. }
\end{aligned}
$$

The effect of these distribution parameters is evident in Fig. 7.

The one-parameter positively skewed distribution by number is given by

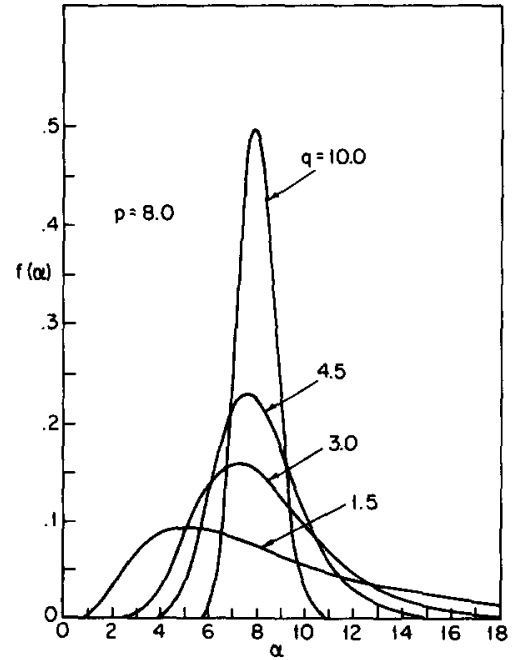

FIG. 7. Effect of the distribution parameters $p$ and $q$ on the log-normal distribution.

$$
f(\alpha)=\frac{3 K^{2} \alpha}{\Gamma(2 / 3)} \exp \left[-(K \alpha)^{3}\right]
$$

where

$\Gamma=$ gamma function,

$K=\lambda / 2 \pi s$,

$s=1.109 w$, and

$w=$ half-width. The half-width is the distance between the two points of the distribution curve at which the frequency is onehalf the value of the modal radius.

Examples of this distribution are given in Fig. 8.

\subsection{Wavelength Associated with $\bar{n}_{0}$}

For particle size distributions with an average particle size $a_{m}$ less than approximately $0.2 \mu \mathrm{m}$, the wavelength exponent evaluated at both $\lambda_{01}$ and $\lambda_{02}$ are essentially equal. In addition, for these small particle sizes, Eq. [22] can be shown to reduce to

$$
\begin{gathered}
\left\{F_{1} \int_{0}^{\infty} \alpha^{3} f(\alpha) n^{*}(\alpha, m) d \alpha\right. \\
\left.-F_{2} \int_{0}^{\infty} \alpha^{3} f(\alpha) P^{*}(\alpha, m) d \alpha\right\} \\
\int_{0}^{\infty} \alpha^{3} f(\alpha) d \alpha
\end{gathered}
$$




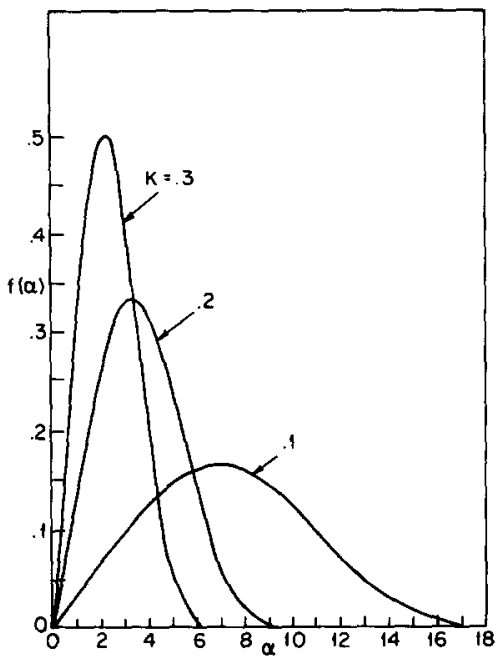

FIG. 8. Effect of the distribution parameter $K$ on the positively skewed distribution.

Therefore, in this case, Eq. [36] can be used directly to solve for the wavelength exponent without the need for numerically solving the integral in the denominator, since it can be inverted analytically, and without the additional complexities of evaluating the following approximation.

When the particle size distribution has an average particle size greater than approximately $0.20 \mu \mathrm{m}$, another approximation can be used. For these larger particle sizes, the turbidity ratio can be determined exactly from two turbidity measurements, but the experimentally observed exponent, computed according to Eq. [10] (see Eq. [39]), can correspond to either wavelength $\lambda_{01}$ or $\lambda_{02}$, or, more than likely, some wavelength between $\lambda_{01}$ and $\lambda_{02}$. Therefore, without any prior knowledge of the wavelength associated with $\bar{n}_{0}$, it seems prudent to use an average exponent $\left\langle\bar{n}_{0}\right\rangle$ defined by

$$
\left\langle\bar{n}_{0}\right\rangle=\frac{\int_{\lambda_{01}}^{\lambda_{02}} \tilde{n}_{0}\left(\lambda_{0}\right) d \lambda_{0}}{\lambda_{02}-\lambda_{01}},
$$

where $\bar{n}_{0}\left(\lambda_{0}\right)$ is given by Eq. [22]. The adequacy of this approximation is indicated by the general agreement between the particle size distributions determined by turbidity analysis and by scanning electron microscopy.

\subsection{Algorithm for Determining Distribution Parameters}

Referring to the turbidity ratio equation, Eq. [20], there are two unknowns if the lognormal distribution is used ( $q$ and $a_{m}$ ), and one unknown if the positively skewed distribution is used $(s)$. Likewise, if only the wavelength exponent equation, Eq. [37], is used the same situation arises. There are two approaches to estimate the unknown distribution:

(1) For the two-parameter distribution, use both Eqs. [20] and [37] (or [36]) simultaneously to solve for the unknown parameters. This approach does not allow any internal check on the turbidity ratio and wavelength exponent techniques.

(2) Use the one-parameter distribution model and solve for the unknown parameter using Eqs. [20] and [37] (or [36]) independently. This approach allows an internal check on the result of each method.

\subsubsection{Two-Parameter Model}

For the log-normal distribution, or any other two-parameter distribution, the solution of Eqs. [20] and [37] (or [36]) for the distribution parameters can be approached by the following graphical and computational methods.

(a) For the optical constants of the system involved ( $m, F_{1}$, and $F_{2}$ ), the turbidity ratio $\left[(\tau \lambda)_{\lambda_{1}} /(\tau \lambda)_{\lambda_{2}}\right]$ is evaluated as a function of $a_{m}$ for the parameter $q$ by numerically integrating Eq. [20]. The results of these calculations are summarized in Fig. 9, where the optical constants are for octacosane $\left(\mathrm{C}_{28} \mathrm{H}_{58}\right)$ particles in water at $25^{\circ} \mathrm{C}$. (See the experimental section for more details on these optical constants and the finite angle of acceptance $\omega$.)

(b) Evaluate the wavelength exponent $\left\langle\bar{n}_{0}\right\rangle$ in the same fashion as the turbidity ratio. The results of these computations are shown in 


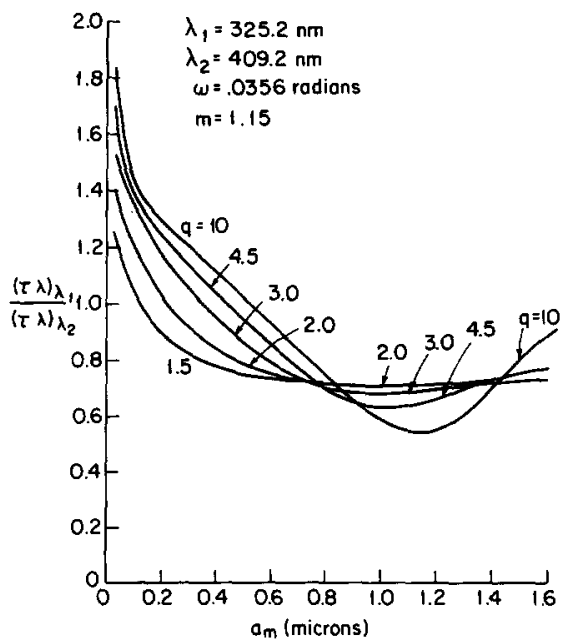

FIG. 9. Turbidity ratio for a log-normal distribution.

Fig. 10 for the same optical constants as in (a).

(c) From the experimentally measured value of the turbidity ratio $\left[(\tau \lambda)_{\lambda_{1}} /(\tau \lambda)_{\lambda_{2}}\right]$, read off the family of $\left(a_{m}, q\right)$ pairs which satisfy Eq. [20] from Fig. 9, or an equivalent figure for different optical constants.

(d) From the experimentally observed value of the wavelength exponent $\left\langle\bar{n}_{0}\right\rangle$, read off the $\left(a_{m}, q\right)$ values which satisfy Eq. [37] from Fig. 10, or an analogous figure.

(e) Steps (c) and (d) result in several $\left(a_{m}\right.$, q) pairs which satisfy Eqs. [20] and [37], sep-

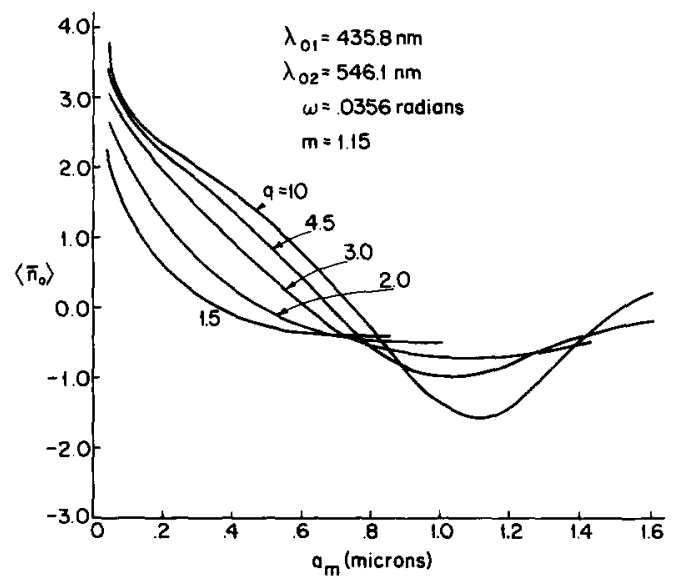

FIG. 10. Wavelength exponent for a log-normal distribution. arately. In order to determine the distribution parameters which satisfy both equations simultaneously, two curves of $q$ versus $a_{m}$ are constructed, one for constant $\left[(\tau \lambda)_{\lambda_{1}} /(\tau \lambda)_{\lambda_{2}}\right]$ and the other for constant $\left\langle\bar{n}_{0}\right\rangle$. Both constant turbidity parameters correspond to the experimentally measured values. The intersection of these two lines gives approximate values of the two distribution parameters, $a_{m}$ and $q$. (Fig. 11 shows this result for distribution 1 , which was used in the experimental section to verify the proposed turbidity technique.)

(f) The approximate values of $a_{m}$ and $q$ can be substituted into Eqs. [20] and [37], and iterated on the computer until the calculated turbidity ratio $\left[(\tau \lambda)_{\lambda_{1}} /(\tau \lambda)_{\lambda_{2}}\right]$ and wavelength exponent $\left\langle\bar{n}_{0}\right\rangle$ agree precisely with the measured values.

\subsubsection{One-Parameter Model}

If the positively skewed distribution, Eq. [35], is used, a scheme similar to the twoparameter model is followed. Using Eqs. [20] and [37], plots similar to Figs. 9 and 10 are constructed with the exception that since there is only the breadth parameter $s$, there will be only a single line for each figure. From the experimentally observed values of the turbidity ratio and wavelength exponent, two independent values of the parameter $s$ can be read directly from these figures. These

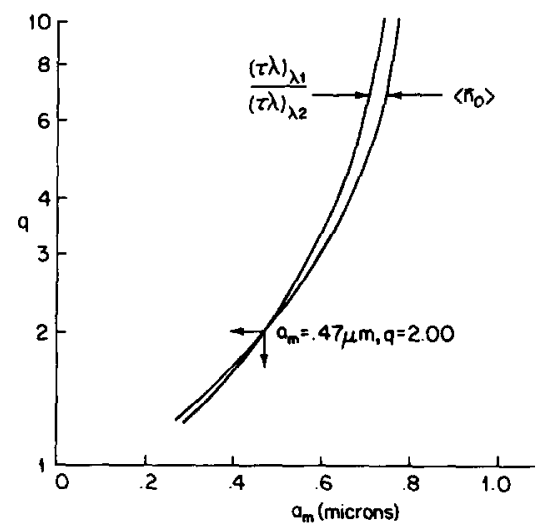

Fig. 11. Graphical determination of log-normal distribution parameters. 
approximate values can be substituted into Eqs. [20] and [37], and iterated on the computer to accurately determine $s$.

\section{EXPERIMENTAL}

\subsection{Preparation of Suspensions}

Polydisperse suspensions were prepared by sonicating deionized water and octacosane $\left(\mathrm{C}_{28} \mathrm{H}_{58}\right)$ at a temperature above the melting point of octacosane $\left(60^{\circ} \mathrm{C}\right)$ and then cooling the resulting emulsion to obtain solid particles. The complete details of this procedure are given by $\mathrm{Li}$ and Fogler (28). By using the ultrasonic method, suspensions of varying degrees of polydispersity can be prepared. Short irradiation times result in very polydisperse systems; while longer irradiation times yield narrow distributions of particle sizes.

The prepared suspensions were diluted to obtain optical density (turbidity) readings and samples for electron microscopic analysis.

\subsection{Electron Microscopy}

As a basis for appraising the turbidimetric method, particle size distributions were also obtained by electron microscopic analysis. By the usual methods of direct count and size measurement on electron micrographs, number density distributions were determined.

\subsection{Optical Constants}

The refractive index and optical dispersion of the particles were obtained from the literature (29). For octacosane particles:

$$
\begin{array}{ll}
\mu_{\mathrm{p}}=1.541 & \left(\lambda_{0}=435.8 \mathrm{~nm} ; 25^{\circ} \mathrm{C}\right), \\
\mu_{\mathrm{p}}=1.531 & \left(\lambda_{0}=546.1 \mathrm{~nm} ; 25^{\circ} \mathrm{C}\right) .
\end{array}
$$

For the water medium (30):

$$
\begin{aligned}
& \mu_{\mathrm{m}}=1.340 \quad\left(\lambda_{0}=435.8 \mathrm{~nm} ; 25^{\circ} \mathrm{C}\right), \\
& \mu_{\mathrm{m}}=1.334 \quad\left(\lambda_{0}=546.1 \mathrm{~nm} ; 25^{\circ} \mathrm{C}\right) .
\end{aligned}
$$

The optical dispersion constants $F_{1}$ and $F_{2}$ were computed from the analytical expressions derived in Appendix B.

\subsection{Instrumentation}

Turbidity measurements were made on a Bausch \& Lomb Spectronic 88 spectrophotometer using the lens-pinhole attachment between the monochromator and the photometer tube. A schematic of the apparatus is shown in Fig. 4.

The monochromatic light beam diverging from the spectrophotometer exit slit $S_{1}$ is partially collimated by a 6.18 -cm focal length lens $L_{1}$ and, after passing through the sample cell, is focused by a $7.30-\mathrm{cm}$ focal length lens $\mathrm{L}_{2}\left(D_{\mathrm{L}_{2}}=1.73 \mathrm{~cm}\right)$ at the pinhole, which has a diameter of $4 \mathrm{~mm}$. Beam stops $B_{1}$ and $B_{2}$ also have 4-mm-diameter holes. Standard 1$\mathrm{cm}$ cells are used in a standard cell holder and carriage. Slit $S_{1}$ is masked to a 4-mm height (normally $16 \mathrm{~mm}$ ) and limited to a maximum width of $0.864 \mathrm{~mm}$.

The resolving power of this arrangement is characterized by the finite angle of acceptance $\omega$. The incident beam diverges from slit $\mathrm{S}_{1}$ 's dimensions to a diameter of $4.5 \mathrm{~mm}$ at beam stop $B_{1}$, and since there is a distance of $38 \mathrm{~mm}$ between $S_{1}$ and $B_{1}$, the source part of the half-angle in the vertical plane is

$$
\theta_{\mathrm{sv}}=\frac{1}{2} \tan ^{-1}\left[\frac{4.5-4.0}{38.0}\right]=0.38^{\circ},
$$

and in the horizontal plane

$$
\theta_{\mathrm{sh}}=\frac{1}{2} \tan ^{-1}\left[\frac{4.5-0.864}{38.0}\right]=2.73^{\circ} .
$$

For rectangular slits, the total source part of the half-angle is determined from (19)

$$
\theta_{\mathrm{s}}=\left[(4 / \pi) \theta_{\mathrm{sv}} \theta_{\mathrm{sh}}\right]^{1 / 2} .
$$

Therefore,

$$
\theta_{\mathrm{s}}=[(4 / \pi)(0.38)(2.73)]^{1 / 2}=1.15^{\circ} .
$$

Since the primary beam is deflected from its original path upon crossing the boundaries 
of the cuvette, the value of $\theta_{\mathrm{s}}$ to be applied to the particles in water at $25^{\circ} \mathrm{C}$ is

$$
\theta_{\mathrm{s}}=\frac{\theta_{\mathrm{s}}^{\prime}}{\mu_{m}}=\frac{1.15}{1.33}=0.86^{\circ} \text {. }
$$

The total detector part of the finite angle of acceptance is computed from Eq. [30]

$$
\theta_{\mathrm{d}}=\frac{\tan ^{-1}(a / f)}{\mu_{m}}=\frac{\tan ^{-1}(2.0 / 73.0)}{1.33}=1.18^{\circ} \text {. }
$$

Therefore, the total angle of acceptance is

$$
\omega=\theta_{\mathrm{s}}+\theta_{\mathrm{d}}=2.04^{\circ} \equiv 0.0356 \text { radians } \text {. }
$$

For the sake of completeness, the minimum diameter of lens $\mathrm{L}_{2}$ required to justify the previous calculations is determined from Eq. [29]. Since the distance between the most remote part of the scattering cell and lens $L_{2}$ is $z=90 \mathrm{~mm}$, the minimum possible diameter of $\mathrm{L}_{2}$ is

$$
D_{\mathrm{L}_{2}} \geq \frac{(2)(2)(90)}{(73)}+4=8.9 \mathrm{~mm} \text {. }
$$

Since the actual diameter is $17.3 \mathrm{~mm}$, this condition is satisfied.

\subsection{Turbidity Measurements}

Because of our instrument's small angle of acceptance, minute irregularities, scratches, etc., on the optical cell also caused a measurable extinction. Therefore, the cells were maintained in a completely fixed position and orientation, and blank values were obtained after filling both the measuring and the reference cell with water.

The aqueous suspensions, $0.04 \%$ by volume, were adjusted in concentration to give optical densities in the range of $0.2-0.8$ at wavelengths of 435.8 and $546.1 \mathrm{~nm}$. The values of the turbidity calculated according to Eq. [2] seldom varied more than 5\% (see Fig. 12); while the computed turbidity ratios varied less than $0.5 \%$ (see Fig. 13). Due to the absence of any significant concentration dependence of the turbidity ratio, the effects of multiple and dependent scattering were negligible. Therefore, extrapolation to zero con-

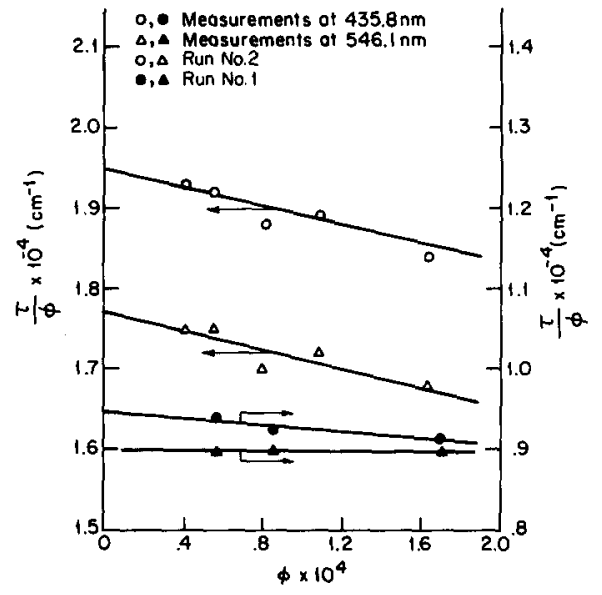

FIG. 12. Normalized turbidity as a function of the volume fraction at wavelengths of 435.8 and $546.1 \mathrm{~nm}$.

centration, the normal procedure for removing these effects, was deemed unnecessary. The logarithmic slope $\left\langle\bar{n}_{0}\right\rangle$ was calculated from

$$
\left\langle\bar{n}_{0}\right\rangle=\frac{\ln \left[\tau_{\lambda_{01}} / \tau_{\lambda_{02}}\right]}{\ln \left[\lambda_{01} / \lambda_{02}\right]}
$$

which is a special case of Eq. [10] for only two turbidity measurements. The slope values are reliable to $1.0 \%$. All measurements were made at $25^{\circ} \mathrm{C}$.

\section{RESULTS}

Distributions from two different suspension samples determined from scanning elec-

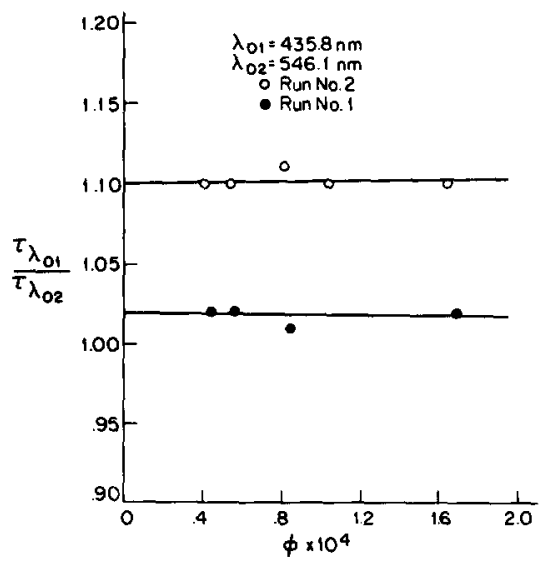

FIG. 13. Turbidity ratio as a function of the volume fraction at wavelengths of 435.8 and $546.1 \mathrm{~nm}$. 


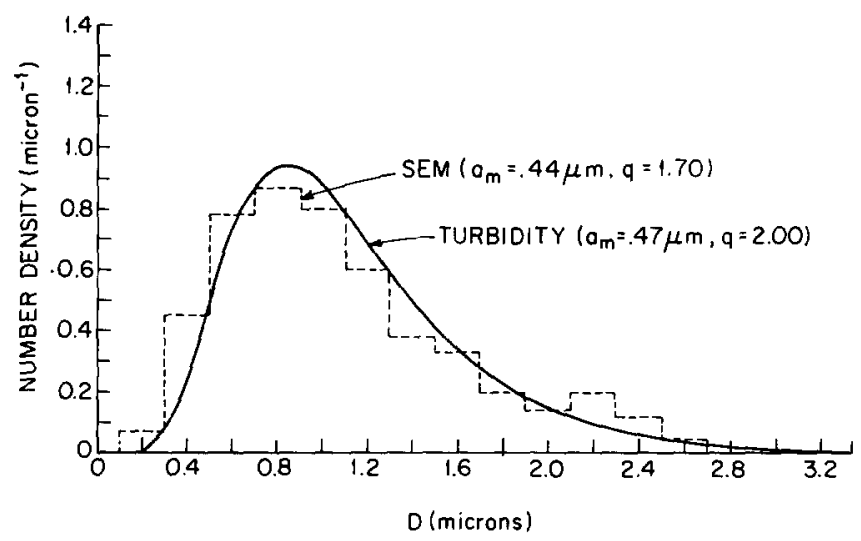

FIG. 14. Comparison of the turbidity method, using the two-parameter log-normal distribution, with SEM results for run 1 .

tron microscopic analysis (SEM) are compared with distributions obtained from the turbidimetric method outlined in section 4 using the log-normal distribution (Figs. 14 and 15 ) and the positively skewed distribution (Figs. 16 and 17). As can be seen from these figures, both distribution models give results which are in agreement with SEM analysis. Since the log-normal distribution is a two-parameter model, a better fit is obtained as compared to the one-parameter positively skewed distribution.

In the turbidity analysis of these distributions, Eqs. [20] and [37] were used. In order to examine the usefulness of Eq. [36], these samples were also analyzed using Eq. [20] and Eq. [36] evaluated at $\left(\lambda_{01}+\lambda_{02}\right) / 2$. For the one-parameter distribution, the results were identical to Figs. 16 and 17; while for the two-parameter distribution, slightly different results were obtained. For run $1, a_{m}$ $=0.49 \mu \mathrm{m}$ and $q=2.5$, and for run $2, a_{m}$ $=0.39 \mu \mathrm{m}$ and $q=2.1$. These findings indicate that the approximation for the wavelength exponent, Eq. [36], may remain valid for particle size distributions with an average size up to approximately $0.50 \mu \mathrm{m}$.

In addition to the accuracy of the proposed turbidity technique, further advantages of the method are the easy, rapid technique and

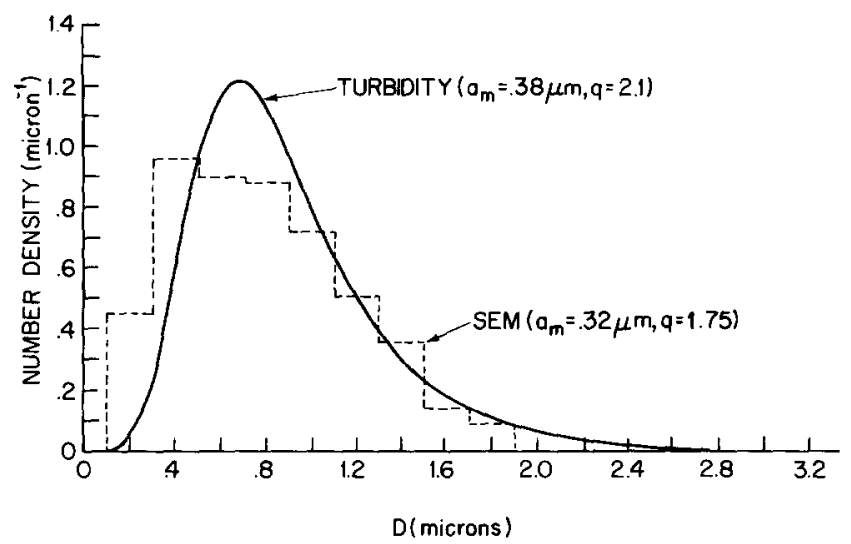

FIG. 15. Comparison of the turbidity method, using the two-parameter log-normal distribution, with SEM results for run 2. 


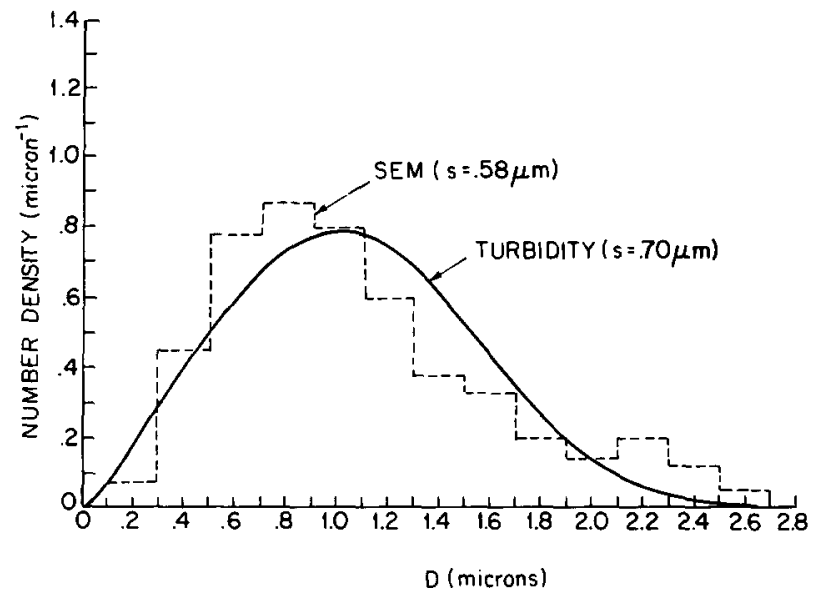

FIG. 16. Comparison of the turbidity method, using the one-parameter positively skewed distribution, with SEM results for run 1.

simple apparatus. The elaborate preliminary calculations are a disadvantage, but the high reproducibility of the results make the method very useful for studying flocculation.

It should be noted that in our experimental analysis we restricted ourselves to the two visible wavelengths available on a conventional ultracentrifuge ( 435.8 and $546.1 \mathrm{~nm}$ ). Even though other wavelengths could have been used, our objective was to demonstrate the direct application of the proposed turbidity technique to determining particle size distributions without modifying the centri- fuge to accomodate other wavelengths. The limitations imposed by using only these two wavelengths are discussed in the following section.

From the above results, one might infer that if three wavelengths were used, one could estimate a three-parameter distribution. Although, in principle, it should be possible to determine all three distribution parameters from turbidity spectra, the work of Nakagaki and Shimoyama (31) has shown that the experimental accuracy required would be excessively high. Therefore, they

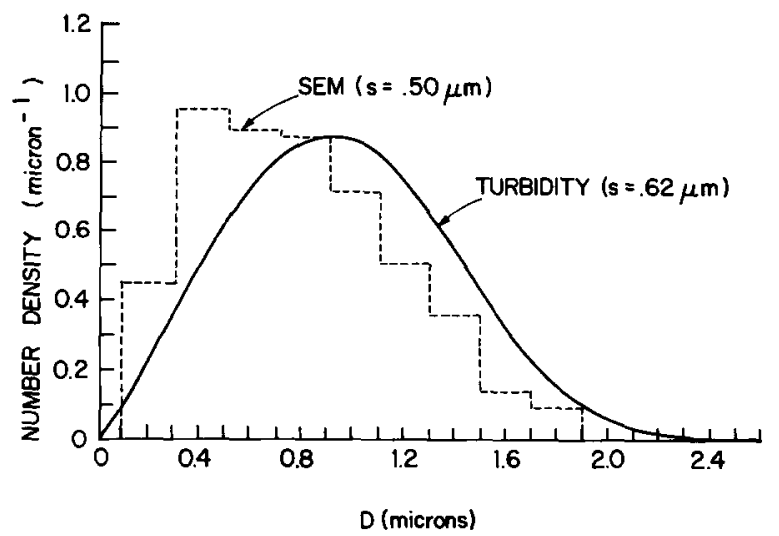

FIG. 17. Comparison of the turbidity method, using the one-parameter positively skewed distribution, with SEM results for run 2. 
recommend using only a one- or a two-parameter distribution.

\section{DISCUSSION}

Even though the proposed turbidity technique can be used to estimate particle size distributions, there are some limitations which should be kept in mind when using this method.

\subsection{Optical Dispersion of the Refractive Index}

On applying the method outlined here, it must be borne in mind that the relative refractive index $m$ varies with wavelength. This has not been taken into account for the theoretical turbidity ratio spectra in Fig. 9. In many instances, such as for the system on which the method has been tested, corrections for the dispersion are not important enough to be applied. In those cases where the dispersion should be taken into account, the turbidity ratio equation, Eq. [20], can reflect this change in refractive index by evaluating the scattering coefficients at the appropriate value of $m$ for each wavelength.

\subsection{Range of Application}

The usefulness of both turbidity ratio and wavelength exponent spectra (within the visible spectral range) for determining size-distribution curves and the sensitivity of the spectra to changes in the degree of polydispersion can be determined with the aid of Figs. 9 and 10. It can be anticipated that the sensitivity to heterodispersion for systems with a geometric mean radius smaller than $0.5 \mu \mathrm{m}$ and with a breadth parameter smaller than $2.1 \mu \mathrm{m}$ will be similar to the systems studied here. While experiments in the lower range of particle diameters and with wider distributions is desirable, the situation which one is bound to find if $a_{m}$ is as small as 0.1 $\mu \mathrm{m}$ can be derived from an inspection of
Figs. 9 and 10. An increase in $q$ from 1.5 to 10 changes both the turbidity ratio and the wavelength exponent spectra just as profoundly no matter whether $a_{m}$ has the value $0.5 \mu \mathrm{m}$ or the value $0.1 \mu \mathrm{m}$. Finally, it should be noted that the method described here, like any other turbidity technique, will, of course, be totally insensitive to particle size distributions if all the particles are sufficiently small compared to the wavelength that Rayleigh scattering applies.

Turbidity spectra will become quite insensitive to particle size distributions if the particles are large relative to the wavelength. Inspection of Figs. 9 and 10 shows that the range of sensitivity terminates for moderately heterodisperse systems $(q=3.0)$ at $a_{m} \simeq 0.8$ $\mu \mathrm{m}$ and for highly polydisperse systems ( $q$ $=2.0$ ) at $a_{m} \simeq 0.6 \mu \mathrm{m}$. Therefore, a system with a distribution breadth parameter $q$ of 2.0 will not respond to the analysis if $a_{m}$ is about $0.8 \mu \mathrm{m}$. In fact, the sensitivity within the visible range of the spectrum begins to fall off seriously if the distribution extends appreciably into the microscopic range.

On being faced with systems in which the particles are too small (Rayleigh range) or too large (microscopic range) to apply both turbidity ratio and wavelength exponent spectra usefully within the visible range, one may, of course, in favorable instances (no true absorption) resolve the problem by making use of spectra in the ultraviolet or intermediate infrared, respectively.

\subsection{Multivalued Solutions}

It is apparent in Figs. 1, 2, 9, and 10, that there exists either a minimum or a maximum in each of these curves and, in addition, there are some extraneous ripples in Fig. 2. These phenomena are a consequence of the sinusoidal nature of the scattering coefficient (see Fig. 3). In this region the various turbidity parameters do not always uniquely define the system; two solutions may be theoretically possible. However, in practice this difficulty 
was not encountered. Referring to Figs. 9 and 10 , the possibility of multivalued solutions can occur for slightly polydisperse systems ( $q=4.5$ ) if $a_{m} \geq 1.0 \mu \mathrm{m}$ and for moderately heterodisperse systems $(q=3.0)$ if $a_{m} \geq 0.8$ $\mu \mathrm{m}$. For highly polydisperse systems $(q=1.5)$, both the turbidity ratio and wavelength exponent spectra remain highly insensitive for $a_{m} \geq 0.55 \mu \mathrm{m}$. Consequently, in this region there is the possibility of thousands of solutions with no way of differentiating the correct distribution from the others.

A detailed study of multivalued solutions by Zollars (32), for the log-normal distribution when $\lambda_{01}=300 \mathrm{~nm}$ and $\lambda_{02}=600 \mathrm{~nm}$ $\left(\lambda_{1}=221 \mathrm{~nm}, \lambda_{2}=450 \mathrm{~nm}\right.$ in a water medium), has shown that as long as the system under investigation has a geometric mean radius in the range $0.03 \mu \mathrm{m} \leq a_{m} \leq 0.94$ $\mu \mathrm{m}$, and a geometric standard deviation in the range $1.05 \leq \sigma_{g} \leq 2.33(1.17 \leq q \leq 20.0)$, there is only one solution. Although Zollars used specific turbidities (Eq. [19]) to determine the distribution parameters, rather than combining turbidity ratio and wavelength exponent measurements, his results are applicable to the present case since both methods are based on the same theoretical foundations.

One notices that as the ratio of the longer to the shorter wavelength increases from 1.26 in the present work to 2.04 in Zollar's study (a $62 \%$ increase), the domain of singular solutions practically doubles. This indicates that, if feasible, one should use two wavelengths that are as far apart as possible.

\subsection{Deviations from Model Distributions}

When using turbidity techniques, there is always the concern that the actual distribution can not be accurately modeled by some theoretical distribution, such as the log-normal distribution. In the present work, run 1 (Fig. 14) is well represented by a log-normal distribution and, consequently, a good fit is obtained throughout the distribution; whereas run 2 (Fig. 15) deviates from a log-normal distribution. The turbidimetrically estimated distribution conforms closely to the central portion of the true distribution while not being strongly biased toward either of the tails. Based on these observations, one can infer that turbidity techniques can be applied to systems which may not conform to the log-normal distribution. The resulting estimated distribution would be a "best-fit" lognormal approximation to the true particle size distribution.

Yang and Hogg (7) have studied this problem using a log-normal distribution on particulate systems that deviate significantly from this type distribution. Their conclusion is the same as the one reached here.

\subsection{Effect of Particle Shape}

An underlying assumption in applying any turbidity technique is that the particles are spherical. Napper and Ottewill $(33,34)$ have examined in detail the deviations obtained between experimental values of the scattering by certain nonspherical particles and the results calculated on the assumption that these particles scatter as equivalent spheres. Silver bromide hydrosols, with a narrow particle size distribution, consisting of either cubes or octahedra were used. In calculating the theoretical turbidities of the cubes, the radius of the equivalent sphere was taken to be half of the face diagonal. For the octahedra, the radius was the distance from the center to a corner.

TABLE I

Estimated Accuracy of Turbidimetric Method

\begin{tabular}{lcccr}
\hline & & \multicolumn{3}{c}{$\begin{array}{c}\text { Average error in calculated } \\
\text { parameters }\left(\%_{0}\right)\end{array}$} \\
\cline { 3 - 6 } \multicolumn{1}{c}{$\begin{array}{c}\text { Measured } \\
\text { quantity }\end{array}$} & $\begin{array}{c}\text { Uncertainty in } \\
\text { measurement (\%) }\end{array}$ & $a_{m}$ & $q$ & $s$ \\
\hline$\left[(\tau \lambda)_{\lambda_{1}} /(\tau \lambda)_{\lambda_{2}}\right]$ & 0.5 & 1.0 & 1.6 & 4.0 \\
$\left\langle\bar{n}_{0}\right\rangle$ & 1.0 & 1.4 & 2.6 & 7.5 \\
$\mu_{\mathrm{p}}$ & 0.3 & 2.5 & 10.1 & 14.3 \\
\hline
\end{tabular}


In both cases, there was quite good agreement between the experimental and computed values of the turbidity. Indeed, this agreement was such as to indicate justification of the various turbidimetric methods described for particles which deviate somewhat from spherical shape.

\subsection{Sensitivity to Experimental Uncertainties}

The accuracy with which the distribution parameters can be determined depends upon the region of the theoretical turbidity curves (Figs. 9 and 10) in which the dispersion falls. In Table I are listed the average errors which can be expected for the probable uncertainties in measurements of the turbidity ratio, the wavelength exponent, and the refractive index of the particles.

\section{SUMMARY}

A turbidimetric technique has been developed to estimate the particle size distribution of nonabsorbing isotropic suspensions in the micrometer to submicrometer range from only two turbidity measurements. For any system of known optical constants and known distributional form, one can use the algorithm outlined to estimate the distribution parameter(s) from observed turbidity ratio and wavelength exponent measurements. The proposed turbidity method has been shown to yield size-distribution curves which are in agreement with scanning electron microscopic analysis.

This technique can be extremely useful for estimating the polydispersity of flocculating emulsions during centrifugation, where previous data analysis methods have failed to account for the formation of aggregates. In addition, since only two turbidity measurements are needed, a conventional ultracentrifuge requires no significant modification of the equipment to apply the proposed turbidity technique.

\section{APPENDIX A: NOTATION}

$a_{m} \quad$ Geometric mean radius for the lognormal distribution, $\mu \mathrm{m}$

$F_{1}, F_{2}$ Optical correction factors to the theoretical wavelength exponent

$f(\alpha) \quad$ Size-distribution function based on number

$m \quad \mu_{\mathrm{p}} / \mu_{m}$

$N \quad$ Total particle concentration, $\mathrm{cm}^{-3}$

$n, n^{*} \quad$ Uncorrected and corrected theoretical Mie wavelength exponent, respectively

$n_{0}, \bar{n}_{0} \quad$ Experimental wavelength exponent for a monodisperse and polydisperse system, respectively

$\left\langle\bar{n}_{0}\right\rangle \quad$ Wavelength exponent averaged over a range of wavelengths

$P, P^{*} \quad$ Uncorrected and corrected Mie lightscattering correction factor to the theoretical wavelength exponent, respectively

$Q, Q^{*} \quad$ Uncorrected and corrected Mie lightscattering coefficient, respectively

$1 /$ ln $\sigma_{g}$, geometric breadth parameter for the log-normal distribution

Particle radius, $\mu \mathrm{m}$

Characteristic parameter of the oneparameter positively skewed distribution, $\mu \mathrm{m}$

$\alpha \quad 2 \pi r / \lambda$, dimensionless particle size

$\lambda \quad \lambda_{0} / \mu_{m}$, wavelength of the primary beam in the dispersing medium, $\mu \mathrm{m}$

$\lambda_{0} \quad$ Wavelength of the incident beam in air, $\mu \mathrm{m}$

Refractive index of the suspending medium; evaluated at $\lambda_{0}$

Refractive index of the particles; evaluated at $\lambda_{0}$

Finite angle of acceptance; equal to one-half the sum of the divergence or convergence of the incident beam and the angle subtended by the detector with ref- 
erence to the center of the scattering volume

$\phi \quad$ Volume fraction of particles

o $2 \alpha(m-1)$, dimensionless particle size

$\sigma_{g} \quad$ Geometric standard deviation for the log-normal distribution

$\theta_{\mathrm{d}} \quad$ Detector's half-angle; equal to onehalf the angle subtended by the detector with reference to the center of the scattering volume

$\theta_{\mathrm{s}} \quad$ Light source's half-angle; equal to one-half the divergence or convergence of the primary beam Turbidity, $\mathrm{cm}^{-1}$

\section{APPENDIX B: ANALYTICAL EXPRESSIONS FOR $F_{1}$ AND $F_{2}$}

The refractive index of water can be accurately calculated from a relation in the International Critical Tables (30)

$$
\mu_{m}=\left[A-B \lambda^{2}+\frac{C}{\left(\lambda^{2}-D\right)}\right]^{1 / 2}+E
$$

where $\lambda=[\mu \mathrm{m}]$. (Note: the subscript 0 has been deleted from $\lambda$ in Eq. [B1] and all other equations in this Appendix. It must be remembered that refractive indices are always evaluated at $\lambda_{0}$.)

At $25^{\circ} \mathrm{C}$ :

$$
\begin{aligned}
& A=1.76148, \\
& B=0.013414, \\
& C=0.0065438, \\
& D=0.013253, \\
& E=0.0001515
\end{aligned}
$$

The refractive index of many hydrocarbons can be correlated by the relationship (6)

$$
\frac{\mu_{\mathrm{p}}^{2}+2}{\mu_{\mathrm{p}}^{2}-1}=a+\frac{b}{\lambda^{2}},
$$

where $\lambda=[\mu \mathrm{m}]$. For octacosane $\left(\mathrm{C}_{28} \mathrm{H}_{58}\right)$ at $25^{\circ} \mathrm{C}$ :

$$
\begin{aligned}
& a=3.3227, \\
& b=-0.026414 .
\end{aligned}
$$

Inserting Eqs. [B1] and [B2] into Eqs. [14] and [15] yields the following analytical expressions for $F_{1}$ and $F_{2}$ :

$$
\begin{aligned}
F_{1}= & 1+\frac{K_{1}}{K_{2}\left[K_{2}+E\right]} \\
F_{2}= & \frac{3 b}{\lambda^{2}\left[K_{3}+2\right]^{1 / 2}\left[K_{3}-1\right]^{3 / 2}\left[K_{2}+E\right]} \\
& +\frac{K_{1}\left[K_{3}+2\right]^{1 / 2}}{K_{2}\left[K_{3}-1\right]^{1 / 2}\left[K_{2}+E\right]^{2}}
\end{aligned}
$$

where

$$
\begin{aligned}
& K_{1}=\lambda^{2}\left[B+\frac{C}{\left(\lambda^{2}-D\right)^{2}}\right], \\
& K_{2}=\left[A-B \lambda^{2}+\frac{C}{\left(\lambda^{2}-D\right)}\right]^{1 / 2},
\end{aligned}
$$

and

$$
K_{3}=a+\frac{b}{\lambda^{2}}
$$

\section{REFERENCES}

1. Meehan, E. J., and Beattie, W. H., J. Phys. Chem. 64, 1006 (1960).

2. Wallach, M. L., Heller, W., and Stevenson, S. F., J. Chem. Phys. 34, 1796 (1961).

3. Wallach, M. L., and Heller, W., J. Phys. Chem. 68, 924 (1964).

4. Wales, M., J. Phys. Chem. 66, 1768 (1962).

5. Gledhill, R. J., J. Phys. Chem. 66, 458 (1962).

6. Walstra, P., J. Colloid Interface Sci. 27, 493 (1968).

7. Yang, R. C., and Hogg, R., Anal. Chem. 51, 758 (1979).

8. Shaw, A. T., and Darling, D. F., "Fourth ParticleSize Analysis Conference," pp. 102-118. Loughborough University of Technology, September 21-24, 1981.

9. Svedberg, The, and Pederson, Kai O., "The Ultracentrifuge." Oxford Univ. Press, London/New York. 1940.

10. Kerker, M., "The Scattering of Light and Other Electromagnetic Radiation." Academic Press, New York, 1969.

11. Heller, W., Bhatnagar, H. L., and Nakagaki, M., J. Chem. Phys. 36, 1163 (1962).

12. Bateman, J. B., Weneck, E. J., and Eshler, D. C., J. Colloid Sci. 14, 308 (1959). 
13. Gumprecht, R. O., and Sliepcevich, C. M., "Tables of Light Scattering Functions for Spherical Particles. Engineering Research Institute Special Publication, University of Michigan, Ann Arbor, Mich., 1951.

14. Pangonis, W. J., Heller, W., and Jacobson, A., "Tables of Light Scattering Functions for Spherical Particles." Wayne State Univ. Press, Detroit, 1957.

15. Heller, W., and McCarty, H. J., J. Chem. Phys. 29, 78 (1958).

16. Walstra, P., Brit. J. Appl. Phys. 15, 1545 (1964).

17. Milne, W. E., "Numerical Calculus." Princeton Univ. Press, Princeton, N. J., 1949.

18. Heller, W., and Tabibian, R. M., J. Colloid Sci. 12, 25 (1957).

19. Walstra, P., J. Appl. Phys. 16, 1187 (1965).

20. Lothian, G. F., and Chappel, F. P., J. Appl. Chem. 1, 475 (1951).

21. Deepak, A., and Box, M. A., Appl. Opt. 17, 2900 (1978).

22. Gumprecht, R. O., and Sliepcevich, C. M., J. Phys. Chem. 57, 90 (1953).
23. Boll, R. H., and Sliepcevich, C. M., J. Opt. Soc. America 46, 200 (1956).

24. Walton, W. H., Trans. Inst. Chem. Eng. 25(Suppl.), 141-142 (1947).

25. "Handbook of Chemistry and Physics," p. F-119. CRC Press Inc., West Palm Beach, Fla, 1959.

26. Deepak, A., and Box, M. A., Appl. Opt. 17, 3169 (1978).

27. Gustav, H., "Small Particle Statistics." Academic Press, New York, 1960.

28. Li, M. K., and Fogler, H. S., J. Fluid Mech. 88, 499 (1978).

29. Johnson, J. F., Ind. Eng. Chem. 46, 1046 (1954).

30. "International Critical Tables," Vol. 7, p. 13. McGraw-Hill, New York.

31. Nakagaki, M., and Shimoyama, T., Bull. Chem. Soc. Japan 37, 1634 (1964).

32. Zollars, R. L., J. Colloid Interface Sci. 74, 163 (1980).

33. Napper, D. H., and Ottewill, R. H., J. Photogr. Sci. 11, 84 (1963).

34. Napper, D. H., and Ottewill, R. H., Trans. Faraday Soc. 60, 1466 (1964). 